\title{
A Front-End Processing Using Subspace-Based Speech Enhancement over Mobile Devices
}

\author{
M. R. L. Daalache, D. Addou, and M. Boudraa
}

\begin{abstract}
The paradigm multi-stream has been shown to result in features combined that can help to increase the robustness of distributed speech recognition (DSR) in the mobile communications. In this paper, we employs a combination of post proceeded Mel-cepstral coefficients (MFCCs) and line spectral frequencies features (LSFs) projected in linear discriminate analysis (LDA) space. The experiments performed on the Aurora 2.0 database using multi-condition training set show that, even with fewer parameters, the proposed front-end provides comparable recognition results to the standard ETSI WI008 advanced front-end, nowadays available in the vocal commands of the GSM mobile communications, while achieving higher accuracy when the signal-to-noise ratio (SNR) is very low.
\end{abstract}

Index Terms-Distributed speech recognition, linear discriminate analysis, multi-stream hidden Markov models, Mel-cepstral coefficients, line spectral frequencies.

\section{INTRODUCTION}

The development of robust speech recognition systems still remains one of the main challenges of many researchers. It has been observed that when modifying a speech recognition system whose models were trained in clean conditions to handle real world environments, its accuracy dramatically degrades [1]. This is mainly due to the mismatches between training and test conditions. To cope with these problems, there are two major approaches. The first approach uses compensation methods to account for the effects of noise and thus, adapt model to new environments [2]. The second approach called front-end process consists of extract robust parameters from corrupted speech prior to pattern matching. A broad range of techniques exists for conveniently representing the speech signal in mismatched conditions [3], [4]. However, most of the current approaches assume that the speech and noise are additive in the linear power domain and the noise is stationary.

The European Telecommunications Standards Institute (ETSI) has established some standards for automatic speech recognition (ASR) feature extraction. In [5], the basic DSR algorithm WI007 Mel-Cepstrum front-end (DSR-FE) is explored. The WI008 Advanced DSR front-end (DSR-AFE) [6] is an extension of the basic DSR-FE; it has been developed

Manuscript received August 24, 2013; revised November 14, 2013.

M. R. L. Daalache is with the University of Technology USTHB, P.O. Box 32 Bab Ezzouar, Algiers, Algeria (Corresponding author, email: mdaalache@usthb.dz)

D. Addou is with the University of Technology USTHB, P.O. Box 32 Bab Ezzouar, Algiers, Algeria (email: daddou@usthb.dz).

M. Boudraa is with Faculty of Electronics and Computer Sciences, FEI, Bab Ezzouar, Algiers, Algeria (email: mboudraa@usthb.dz). in order to improve the basic front-end robustness for environmental noisy speech. The DSR-AFE uses two-stage Mel-warped Wiener filtering and an SNR-dependent waveform processing to improve the SNR of speech. The resulting speech signal is further processed into MFCCs. Finally a blind equalization is applied to the cepstral coefficients. The DSR-AFE represents the state-of-art in terms of recognition accuracy for noisy speech but it suffers from problem of high computation load due to the use of double Wiener filtering.

In the last decade, some studies were carried out on the combination of robust acoustic features. It has been shown the effectiveness of merging different sources of information about the speech signal that could be lost when using only the MFCCs to recognize uttered speech. In previous paper, we introduced a multi-stream paradigm for DSR in which, we combine MFCC with LSF features. These features are adequately transformed and reduced in multi-stream scheme using Karhunen-Loeve Transform (KLT) [7]. The results obtained have shown a significant improvement in the recognition performance of the first ETSI standard. In present work, we investigate different methods to enhance parameters corrupted by the noise effect. Indeed, one effective way to reduce effect of noise in robust speech recognition is to proceed directly on corrupted parameters. We start by extract standard MFCCs with their deltas on which we apply noise reduction methods. Finally LSF parameters projected in LDA space are concatenated with processed MFCCs and delta MFCCs to constitute robust multi-stream feature vector. The latter is then used to train multi-stream hidden Markov models (HMMs). The reason that motivated us to consider LSFs is related to the fact that LSF regions of the spectrum may stay above the noise level even in very low SNR ratio, while the lower energy regions will tend to be masked by the noise energy [8]. Moreover, the extraction LSF parameters can be done as part of the process of MFCCs calculation which allows reducing significantly the added computation load.

The outline of this paper is as follows. In Section II, we describe the proposed front-end. In Section III, we proceed with a description of the multi-stream statistical framework and Section IV is devoted to the description of the evaluation experimental of our proposed approach and the corresponding discussion. Finally, a conclusion will be presented in the last Section.

\section{FRONT-END DESCRIPTION}

There is a wide range of methods that can be used to parametrically represent speech signal. The most popular of them are MFCCs, PLPs (perceptual linear predictive), LPCs 
(linear predictive coding), LSFs and so on. However, if we consider a signal corrupted by an additive or convolutional noise, these methods become less efficient. Often, the degradation of performance is minor when the SNR is high and more important at low SNR levels. So it is challenging to investigate a new method that provides robust and reliable parameters. In this work, we propose a scheme of features enhancement methods applied over MFCC and LSF parameters. We start by using a 3-streams feature vector. The First two streams are dedicated to the MFCCs and their first derivatives. We apply cepstral mean compensation (CMC) and cepstral variance normalization $(\mathrm{CVN})$ over the first two streams in order to reduce MFCCs distortion in presence of additive and convolutional noises. Essentially, CMC consists of subtracting, from each frame, the cepstral mean, calculated across the utterance. For instance, we have an utterance represented data by a matrix data $\mathrm{C}$ of element $C_{t d}$ whose each row represents a feature vector and each column represents a time sequence. Thus, $\mathrm{CMC}$ and $\mathrm{CVN}$ are applied as follow:

$$
C_{t d}^{\prime}=\left(C_{t d}-\mu_{d}\right) / \sigma_{d}
$$

There $C_{t d}$ is $\mathrm{d} t h$ multi-stream coefficient of the $t h$ frame. $\mu_{d}$ and $\sigma_{d}$ are respectively the mean and variance of $\mathrm{d} t h$ time sequence coefficient. Each mean-subtracted and variance-normalized time sequence is further processed by an autoregressive moving average (ARMA) filter [9]. Indeed, ARMA filter was used for smoothing out any spikes in the time sequence that might be caused by noise. Finally, LSF

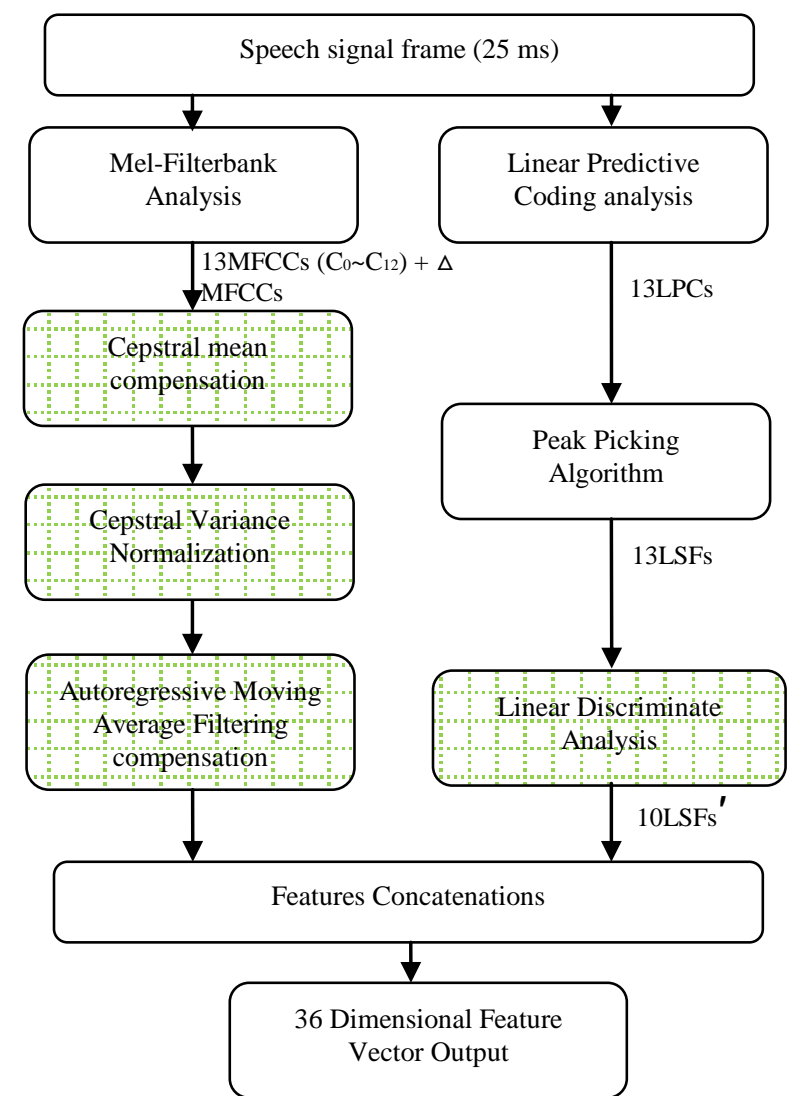

Fig. 1. Bloc diagram of proposed front-end (add processing blocks related to noise reduction effects are highlighted). parameters projected in LDA space (LSFs') are used as the elements of the third stream vector. LDA is applied to preserve the most descriptive information by eliminating the noisy principal components and reduce the dimension of the features [10]. A block diagram of the proposed front-end is shown in Fig. 1.

\section{Multi-STREAm FrameWORK}

The HMMs are often used trough parameters estimation of a Gaussian set to model the statistical variations of speech [11], [12]. A HMM model is basically a stochastic finite state automaton which generates an observation string produced as a result of an emitting vector $O_{t}$ for each transition from state $i$ to $j$. The output distribution associated with each state is dependent on one or more distinct stream which must be statistically independent. For example, static and dynamic coefficients correlation is small. Hence, as coefficients are generated by independent sources, we have to putting them in separate streams in order to obtain a better modelisation by HMMs.

After, a multi-stream features vector formed by concatenated these streams is used to train the corresponding phone (or word) HMM.

Consider an observation sequence $O, O=O_{1} \ldots O_{t} \ldots O_{T}$, possibly representing the utterance to be recognized. In this sequence each vector $O_{t}$ is composed of $S$ input streams. At the time $t$, the probability $b$ of observing a vector $\mathrm{O}$ in state $\mathrm{j}$ is given by:

$$
b_{j}\left(O_{t}\right)=\prod_{s=1}^{S}\left[b_{j s}\left(O_{s t}\right)\right]^{\gamma_{j s}}
$$

where $b_{j s}\left(O_{s t}\right)$ is the probability of observing a vector of stream $s$ at the time $t . \gamma_{j s}$ represents the stream weight and specifies the contribution of each stream to the overall distribution. It is normally assumed to satisfy the following constraints:

$$
0 \leq \gamma_{j s} \leq 1 \text { and } \sum_{s}^{S} \gamma_{j s}=1
$$

The distribution associated with each stream $s$ is represented by a multivariable mixture Gaussian density:

$$
b_{j s}\left(O_{s t}\right)=\sum_{m=1}^{M} C_{j m} N\left(O_{s t} ; \mu_{j s m} ; \sum_{j s m}\right)
$$

$M$ is the number of mixture components, $C_{j s m}$ is the $m t h$ mixture weight of state $j$ for the stream $s . N$ denotes a multivariable Gaussian having $\mu$ as the mean vector and $\sum$ as the covariance matrix that is:

$$
\begin{aligned}
& N\left(O_{s t} ; \mu_{j s m} ; \sum_{j s m}\right)=\left(1 / \sqrt{2 \pi^{n}\left|\sum_{j s m}\right|}\right) \\
& \quad \exp -\left((-1 / 2)\left(O_{s t}-\mu_{j s m}\right)^{\prime} \sum_{j s m}^{-1}\left(O_{s t}-\mu_{j s m}\right)\right)
\end{aligned}
$$


To investigate the multi-stream paradigm using the proposed features for DSR, we have performed a several experiments combining multiple sources of information about the speech signal that could be lost when using only the cepstral analysis. Recall that the proposed multi-stream has three streams for all HMM states. The first stream consists of classical MFCCs plus cepstral coefficient $C_{0}$. The second stream includes MFCC delta coefficients and the delta $C_{0}$. Both streams are processed by CMC, CVN and ARMA filter. The third stream contains LDA projected LSFs. Although stream weights may influence reconnaissance accuracy, we often assign the same weight set to whole states in order to reduce system complexity

\section{EXPERIMENT AND EVALUATION}

To examine the efficiency of the proposed front-end for continuous speech recognition, a series of experiments on Aurora 2.0 database are performed.

\section{A. Database Description}

Submit Aurora 2.0 database is based on version of Tidigit down-sampled from $20 \mathrm{kHz}$ to $8 \mathrm{kHz}$, and consists of a connected digits task spoken by American English talkers [13]. 8440 utterances provided from 55 male and 55 female adults and filtered with the G.712 (GSM standard) characteristics are used for training task [14]. Two training modes have been defined. The first uses only clean utterances in training task. The second mode is based on the usage of clean and noisy data. The latter mode, referred as multi-condition training set, contains utterances with 4 different noise types (subway, train, babble, car and exhibition hall) recorded at 5 SNR levels (5 to $20 \mathrm{~dB}$ and "clean"). Thus, a total of $4 \times 5$ subsets are available. Each subset consists of 422 utterances.

Three different tests set A, B and C are defined. They contain data artificially distorted by adding recorded noises at different SNR. Speech and noise are with the G.712 before being added. In test set A, four distinct noise signals (subway, train, babble, car and exhibition hall) were added to clean case at SNRs of $-5,0,5,10,15$ and $20 \mathrm{~dB}$. The clean case is considered as subset test. A total of $4 \times 7 \times 1001=28028$ utterances will be used.

Test set B has the same organization as in A. However it uses four different noises, namely restaurant, street, airport and train station.

Test set $\mathrm{C}$ consists of only $2 \times 7$ subsets containing one noise type of test set A and one noise type of test set B. Speech and noise are filtered with a MIRS characteristic before being added at SNRs of $20 \mathrm{~dB}, 15 \mathrm{~dB}, 10 \mathrm{~dB}, 5 \mathrm{~dB}, 0 \mathrm{~dB}$ and $-5 \mathrm{~dB}$. The clean case is also considered in test. This test set is supplied with database to show the influence of distortions related to transmission channel on recognizer robustness.

\section{B. Features Combination}

In our experiments, we start by using 39-dimensional multi-stream feature vectors consisting of 13 cepstral features ( $C_{0}$ to $C_{12}$ ), their first derivatives and $13 \mathrm{LSF}$ coefficients. The first two streams are processed by CMC, CVN and ARMA filter in order to improve MFCCs robustness. The dimension of LSFs is reduced from 13 to 10 using LDA. Finally, we obtain 36-dimensional multi-stream feature vectors.

16 states whole-word HMMs were used to model each digit of Aurora 2.0 multi-condition training set. A single HMM consisting of 3 states is used to model the pauses. Note that, the advantage of using multi-conditional training mode is the possibility of considering the noise characteristics as part of the word models, especially for pause model. Training and recognition were carried out by HTK toolkit [15].

\begin{tabular}{|l|l|c|c|c|c|c|c|c|c|} 
TABLE I. RECOGNITION RATE (\%) OF THE BASIC DSR SYSTEM AND THOSE USING THE MULTI- VARIABLE ON TEST A, B AND C OF AURORA DATABASE \\
\begin{tabular}{|l|l|c|c|c|c|c|c|c|}
\hline Noise type & Multi-variable vector & clean & $\mathbf{2 0 d B}$ & $\mathbf{1 5 d B}$ & $\mathbf{1 0 d B}$ & $\mathbf{5 d B}$ & $\mathbf{0 d B}$ & $\mathbf{- 5 d B}$ \\
\hline \multirow{2}{*}{ Subway } & DSR-FE (39) & 98.68 & 97.61 & 96.47 & 94.44 & 88.36 & 66.9 & 26.13 \\
(Test A) & DSR-AFE (39) & $\mathbf{9 9 . 1 7}$ & $\mathbf{9 8 . 7 1}$ & 97.7 & 95.27 & 91.5 & 77.62 & 45.07 \\
& Proposed (36) & 98.65 & 98.31 & $\mathbf{9 7 . 8 5}$ & $\mathbf{9 6 . 4 7}$ & $\mathbf{9 2 . 0 8}$ & $\mathbf{7 8 . 4 5}$ & $\mathbf{4 7 . 1 9}$ \\
\hline \multirow{2}{*}{ Restaurant } & DSR-FE (39) & 98.68 & 96.87 & 95.3 & 91.96 & 83.54 & 59.29 & 25.51 \\
(Test B) & DSR-AFE (39) & $\mathbf{9 9 . 1 7}$ & $\mathbf{9 8 . 4 3}$ & $\mathbf{9 7 . 7 6}$ & 95.49 & 88.61 & $\mathbf{7 0 . 4 9}$ & $\mathbf{3 2 . 7 6}$ \\
& Proposed (36) & 98.65 & 98.04 & 97.73 & $\mathbf{9 5 . 7}$ & $\mathbf{8 9 . 1 3}$ & 69.73 & 32.24 \\
\hline \multirow{2}{*}{ Subway } & DSR-FE (39) & 98.5 & 97.3 & 96.35 & 93.34 & 82.41 & 46.82 & 18.91 \\
(Test C) & DSR-AFE (39) & $\mathbf{9 9 . 1 1}$ & 98.5 & 97.82 & $\mathbf{9 6 . 1 3}$ & 90.54 & 72.37 & 36.51 \\
& Proposed (36) & 98.89 & $\mathbf{9 8 . 5 6}$ & $\mathbf{9 7 . 8 8}$ & 95.86 & $\mathbf{9 1 . 3 4}$ & $\mathbf{7 4 . 9 1}$ & $\mathbf{4 1 . 1 1}$ \\
\hline
\end{tabular}
\end{tabular}

\section{Test Results and Discussion}

Performances of the proposed front-end were compared with those of the ETSI front-ends for the three test sets. Table 1 gives the results for GSM speech corrupted by different types of test noise, namely subway for test A and C, and restaurant for Test B. Best results in terms of word recognition accuracy are edited in bold. It can be observed that the proposed front-end performs much better than the standard DSR-FE, Moreover, it provides comparable recognition performance compared with the reference DSR-AFE. Furthermore we have to be considering that the DSR-AFE has been developed over years to achieve an optimal error rates on the Aurora 2.0 task. Thus, it does not necessarily reach higher performances for other database.

For very low SNRs, when the SNR decreases less than $5 \mathrm{~dB}$, the use of LSF front-end with 36-dimensionnal feature vector leads to a significant improvement in word recognition accuracy (Fig. 2). We note that our approach is all the better, when the SNR decreases. The substituting of acceleration components and energy by the LSFs in the baseline vector led to an improvement in rate recognition with a consequent dimension vector. Note that, in this Fig. 2, the (C) following the names of a noise type refer to noisy speech data from test set C. 
On the other hand, both the proposed front-end and the advanced front-end perform much better than the ETSI standard MFCC front-end, particularly in the noisier conditions. In some cases, more than double of the recognition accuracy can be achieved by using the proposed front-end.

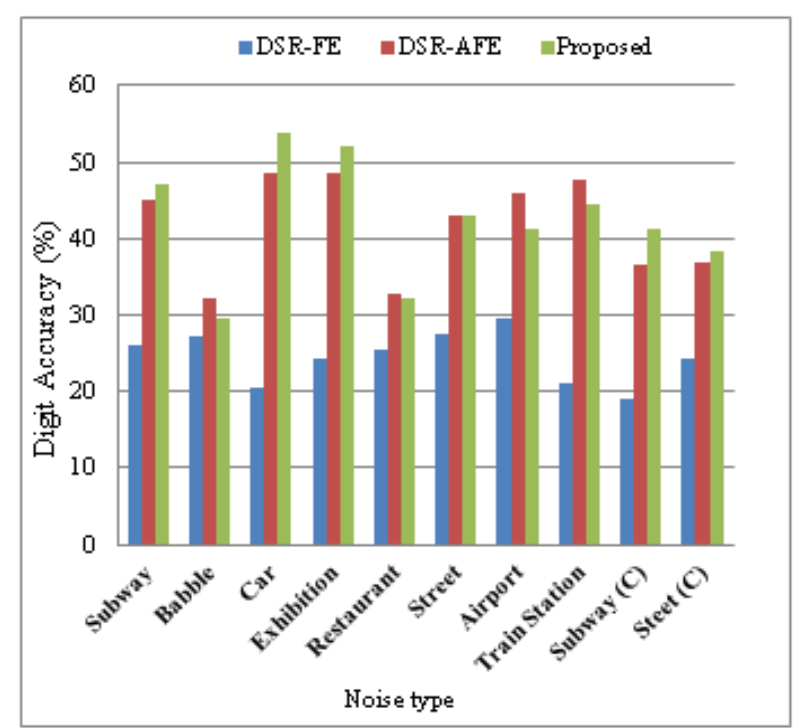

Fig. 2. Recognition results for whole Aurora test sets, proposed front-end compared with ETSI front-ends by noise type at $\mathrm{SNR}=-5 \mathrm{~dB}$.

\section{CONCLUSION}

In this study, we have proposed a new robust front-end as an alternative for the ETSI DSR advanced front-end codec. This approach uses feature enhancement techniques performed over LSF and MFCC parameters. The parameters proceeded are then combined in order to improve the performance of DSR in severely degraded environments. Experimental results on the Aurora 2.0 database revealed the effectiveness of this front-end. Indeed, the latter is found to obtain a high score of error rate reduction compared to the standard DSR-FE. Moreover, the proposed front-end provides comparable DSR-AFE recognition accuracy with fewer parameters over whole tests set. The robustness of our front-end is better under severely degraded noise conditions (i.e. $-5 d B$ ).

Although, extracting other parameters seems to increase computational complexity of the proposed front-end.

However, recall that the main drawback of the DSR-AFE is its high computation load due its noise reduction bloc $\mathrm{d}$ and the extraction LSF parameters can be done as part of the process of MFCCs calculation which allows reducing significantly the added computation load. Thus, the proposed front-end appears to be more appropriate compared to other ETSI standards for applications running on handheld devices with limited memory and processing power. In future works, we will propose solutions to reach optimal weight stream values in order to improve the speech recognition accuracy. Indeed, as seen in (2), we can notice that, for multi-stream vector, the recognition depends on weighted stream parameters.

\section{REFERENCES}

[1] Y. Gong, "Speech recognition in noisy environments: A survey," Speech Communications, vol. 16, no. 3, pp. 261-291, April 1995.

[2] H. G. Hirsch, "Automatic speech recognition in adverse acoustic conditions," Advances in Digital Speech Transmission, John Wiley \& Sons, Ltd, 2008, ch. 16.

[3] J. C. Junqua and J. P. Haton, Robustness in Automatic Recognition, Kluwer Academic Publishers, Springer-Verlag, New York, 1995.

[4] R. Rose and P. Momayez, "Integration of multiple features sets for reducing ambiguity in automatic speech recognition," in Proc. IEEE-ICASSP, vol. 4, pp. 325-328, April 2007.

[5] ETSI, "Speech processing, transmission and quality aspects distributed speech recognition; front-end feature extraction algorithm, compression algorithms," report of ETSI ES 201 108, 2000.

[6] ETSI, "Speech processing, transmission and quality aspects; distributed speech recognition; advanced front-end feature extraction algorithm; compression algorithm," report of ETSI ES 202 050, 2002.

[7] D. Addou, S. A. Selouani, M. Boudraa, and B. Boudraa, "Transform-based multi-features optimization for robust distributed speech recognition," in Proc. GCC Conference and Exhibition (GCC) IEEE, Dubai UAE, 2011, pp. 505-508.

[8] D. Addou, S. A. Selouani, K. Kifaya, M. Boudraa, and B. Boudraa, "A noise-robust front-end for distributed speech recognition in mobile communications," Int. J. Speech Technology, vol. 10, no 4, pp 167-173, March 2009

[9] C. P. Chen, J. Bilmes, and K. Kirchhoff, "Low-resource noise-robust feature post-processing on Aurora 2.0," in Proc. 7th Int. Conf. Spoken Language Processing, Colorado, 2002, pp. 2445-2448.

[10] R. Haeb-Umbach, D. Geller, and H. Ney, "Improvements in connected digit recognition using linear discriminant analysis and mixture densities," in Proc. IEEE-ICASSP, vol. 2, pp. 239-242, April 1993.

[11] L. Rabiner and B.-H. Juang, Fundamentals of Speech Recognition. Englewood Cliffs, NJ, Prentice-Hall, 1993.

[12] A. Norouzian, "Incorporation of multiple acoustical features in to DSR systems," Master thesis, INRS-EMT, Quebec University, 2008.

[13] H.G. Hirsch and D. Pearce, "The Aurora experimental framework for the performance evaluation of speech recognition systems under noisy conditions," in Proc. ISCA ITRW ASR200, France, 2000.

[14] ITU recommendation G. 712, Transmission performance characteristics of pulse code modulation channels, November 1996.

[15] S. J. Young, "HTK version 3.4: Reference and User Manual," Cambridge University, 2009.

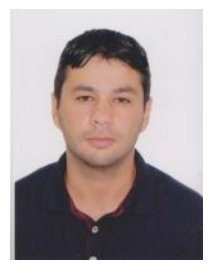

M. R. L. Daalache was born on November 20, 1987 in Algiers, Algeria. He received the B. S. and M. S. degree from the University of Sciences \& Technology Houari Boumediene, Algiers, Algeria.

In 2011, he joined the Spoken Communication and Signal Processing Laboratory in Algiers. Since then, his study interest focused on machine learning, speech recognition and understanding, speaker recognition and information extraction.

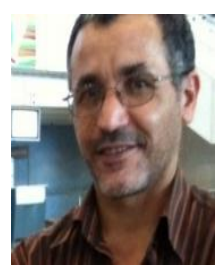

D. Addou was born on November 20, 1987 in Algiers, Algeria. He received the Magister Degree from the Department of Electronic at School of polytechnic Algiers in year 1992. He completed Doctorat in signal processing and speech communication from the Departement Telecommnication at the computer and electronical faculty in Algiers Universitys.

$\mathrm{He}$ is a senior lecturer at the Faculty of Computer and Electronical Sciences at Technology Houari Boumediene University and his main research interests are signal processing, speech communication and Robustess mobile communication system.

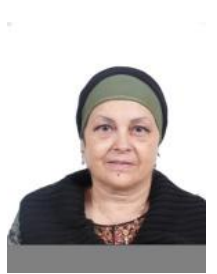

M. Boudraa was born on April 1, 1950 in Algiers, Algeria. She received the electronic engineering diploma in 1978 and Magister degree in 1984 from Algiers High polytechnic school and $\mathrm{PhD}$ degree in 2000 from Algiers H.B. Sc. and Tech. University. She is lecturer since 1978.

She has been involved in many ministery projects. Her current research interests include speech recognition, audio compression, speech communications, acoustics and digital signal processing 